\title{
Charging energy and barrier height of pentacene on Au(111): A local-orbital hybrid-functional density functional theory approach
}

\author{
B. Pieczyrak, ${ }^{\text {a) }}$ E. Abad, F. Flores, ${ }^{\text {b) }}$ and J. Ortega \\ Departamento de Física Teórica de la Materia Condensada, Universidad Autónoma de Madrid, \\ ES-28049 Madrid, Spain
}

(Received 4 June 2011; accepted 29 July 2011; published online 24 August 2011)

\begin{abstract}
We analyze the pentacene/Au(111) interface by means of density functional theory (DFT) calculations using a new hybrid functional; in our approach we introduce, in a local-orbital formulation of DFT, a hybrid exchange potential, and combine it with a calculation of the molecule charging energy to properly describe the transport energy gap of pentacene on Au(111). Van der Waals forces are taken into account to obtain the adsorption geometry. Interface dipole potentials are also calculated; it is shown that the metal/pentacene energy level alignment is determined by the potential induced by the charge transfer between the metal surface and the organic material, as described by the induced density of interface states model. Our results compare well with the experimental data. (C) 2011 American Institute of Physics. [doi:10.1063/1.3626522]
\end{abstract}

\section{INTRODUCTION}

Organic semiconducting materials have recently attracted much attention due to their unique properties for the prospective applications in organic electronics, such as LED, photovoltaic cells, and field effect transistors. Among the most commonly used organic semiconductors, pentacene $\left(\mathrm{C}_{22} \mathrm{H}_{14}\right)$ is one of the most promising materials due to its high fieldeffect mobility. ${ }^{1}$ The performance of these organic devices depends crucially on the different energy barriers that control the carrier transport between the layers. These barriers are determined by the relative positions of the molecular levels across the metal-organic (MO) or the organic-organic interfaces. ${ }^{2,3}$ Molecular level alignment at organic junctions has been widely investigated in the last decade. Since the Schottky-Mott limit (where the vacuum level alignment rule has been used) was disproved, ${ }^{4,5}$ several mechanisms have been proposed to explain the barrier formation at MO interfaces: interface chemical reaction and the formation of gap states in the organic material, ${ }^{6-9}$ orientation of molecular dipoles, ${ }^{10,11}$ or compression of the electron metal tails due to the Pauli repulsion ("pillow effect"). ${ }^{8}{ }^{12-14}$ It has also been suggested that the tendency of the charge neutrality level (CNL) of the organic material to align with the interface Fermi level ${ }^{15,16}$ plays also an important role; this mechanism is associated with the induced density of interface states (IDIS) and the charge transfer between the two materials. More recently, this model has been extended to include the Pauli repulsion and intrinsic molecular dipoles in the unifiedIDIS model. ${ }^{14,17}$ While in the IDIS model the mechanism associated with the barrier formation is mainly due to the rearrangement of charge at the interface, in the integer charge transfer model ${ }^{18}$ polaronic states in the organic material are assumed to modify the transfer mechanism, creating spontaneous integer charge transfer at the interface.

\footnotetext{
a)Electronic mail: barbara.pieczyrak@uam.es.

b) Electronic mail: fernando.flores@uam.es.
}

In this paper, we consider the case of a pentacene/ $\mathrm{Au}(111)$ interface and analyze its barrier height for a single molecule and a full monolayer (see Fig. 1); in our calculations, we have assumed to have one of four predominant experimental unit cells: $2 \times \sqrt{39}$ structure [Fig. 1(a)]; we use this one because at lower coverages pentacene molecules tend to have larger spacings between the rows of molecules such as in the chosen type..$^{19}$ Other authors have analyzed theoretically the $6 \times \sqrt{7}$ geometry using the density functional theory (DFT) techniques within generalized gradient approximation (GGA); ${ }^{20}$ for the sake of comparison, we have also calculated this geometry [Fig. 1(b)]. We reconsider this interface because neither standard local-density approximation (LDA) nor GGA-DFT approximations yield an appropriate description of the organic transport energy gap..$^{21,22}$ In particular, the Kohn-Sham (KS) gap, calculated as the difference between the eigenvalues of the lowest unoccupied molecular orbital (LUMO) and the highest occupied molecular orbital (HOMO), is too small (either using LDA or GGA). It has been argued elsewhere ${ }^{23,24}$ that the effective charging energy of the molecule, $U$, can be used to correct the Kohn-Sham energy gap, $E^{\mathrm{KS}}$, to yield the following transport gap:

$$
E^{t}=E^{\mathrm{KS}}+U,
$$

an equation that will be used below to determine $E^{t}$ self-consistently.

In our approach, we calculate the charging energy $U$ for a pentacene molecule on $\mathrm{Au}(111)$ by means of DFT calculations; then, we obtain the appropriate transport gap (see Eq. (1)) introducing in the DFT calculation a hybrid potential that mixes a fraction of Hartree-Fock (HF) exchange potential with the "local" exchange potential of the molecule, using for this purpose a local-orbital formulation of DFT. We also analyze the interface dipoles and discuss the barrier height formation as a function of the metal work-function. We interpret our calculations within the unified-IDIS model and show how our results can be understood in terms of the molecule 
$\mathrm{CNL}$ and a screening parameter, $S$, operating at the interface level. ${ }^{22}$ In Sec. II, we first present our calculational approach introducing the hybrid HF-DFT method. We also discuss the pentacene/Au interaction energy correcting our LDA calculation by including van der Waals forces: this will be shown to affect the pentacene/Au equilibrium distance, enlarging a little the value given by a DFT-LDA calculation. In Sec. III, we present our hybrid-potential DFT results for a single pentacene molecule on $\mathrm{Au}(111)$ and a monolayer coverage, including charging energy effects, and we discuss the pillow and metal surface dipole corrections to our DFT-LDA results. Finally, in Sec. IV, we present our conclusions.

\section{PENTACENE/AU INTERACTION: METHOD OF CALCULATION, DFT, HYBRID POTENTIAL, AND VAN DER WAALS CORRECTION}

\section{A. Method of calculation}

We analyze the pentacene/Au(111) interface using a similar approach to the one presented in Ref. 25 for benzene. A summary of the main points of this calculation is as follows:

(a) In a first step, we perform FIREBALL calculations for the pentacene/Au(111) system; FIREBALL (Ref. 26) is a real-space LDA-DFT code that uses a basis set of numerical atomic orbitals (NAOs) that are strictly zero beyond a given cut-off radius (the FIREBALL orbitals ${ }^{27}$ ) and that is based in a self-consistent implementation of the Harris-Foulkes functional. ${ }^{28}$ Due to this combined use of short-range NAOs and a selfconsistent version of the Harris-Foulkes functional, in this technique, four-center electron-electron integrals are not required during the calculation, while the two-center and three-center interactions can be precalculated on a numerical grid and stored in data tables. The specific values needed during the simulation are obtained using a fast tabulation-interpolation scheme, ${ }^{27}$ resulting in a computationally very efficient approach. In these calculations, we use a minimal basis set of $s p^{3} d^{5}$ NAOs for $\mathrm{Au}, s p^{3}$ for $\mathrm{C}$, and $s$ for $\mathrm{H}$ with the following cut-off radii (in a.u.): $s=4.5$, $p=4.9, d=4.3(\mathrm{Au}) ; s=4.5, p=4.5(\mathrm{C})$; and $s=4.1(\mathrm{H})$. For Au, this basis set yields a bulk gold lattice parameter of $4.12 \AA$ versus an experimental one of $4.07 \AA .{ }^{29}$ For pentacene, we have used the minimal $s p^{3}$ basis set of optimized NAOs; ${ }^{30}$ this yields a range of values between 1.36 and $1.47 \AA$ for the $\mathrm{C}-\mathrm{C}$ nearest neighbours distance, to be compared with the experimental values of 1.35-1.45 $\AA^{31}$ (in GGA-DFT these distances are 1.38-1.46 ̊). ${ }^{32}$ This pentacene minimal basis set yields a Kohn-Sham energy gap of $1.6 \mathrm{eV}$, to be compared with $1.1 \mathrm{eV}$ for a converged basis set GGA-DFT calculation. ${ }^{32}$ The main inaccuracy of this minimal basis approach appears in the initial relative alignment of the electronic levels of the metal and pentacene; this problem is corrected in our approach using a "shift operator" for the pentacene levels as discussed in Ref. 25. As for the gas-phase molecule, the ionization energy is $6.6 \mathrm{eV}$ (Ref. 33) and $E^{t}=5.2 \mathrm{eV},{ }^{34}$ the pentacene mid-gap is located $1.2 \mathrm{eV}$ above the metal work-function.

(b) In a LDA-DFT (or GGA-DFT) approach, the KohnSham energy gap is too small (see Eq. (1)); in the gas-phase pentacene, $E^{\mathrm{KS}}=1.1 \mathrm{eV}(1.6 \mathrm{eV}$ in our basis set) and $E^{t}=5.2 \mathrm{eV}^{34}$ For pentacene on $\mathrm{Au}(111)$, $E^{t}$ is reduced due to surface polarization effects; we determine $E^{t}$ analyzing the case of a single molecule on the surface. In our approach, we first obtain $U$ by means of the equation: $U=e V^{\mathrm{IDIS}} / n,{ }^{23} V^{\mathrm{IDIS}}$ being the potential induced in the molecule due to the metal-molecule transfer of charge (see below) and $n$ the number of electrons transferred from the metal to the molecule. Then, the energy level spectrum is corrected, according to the energy gap value given by Eq. (1), introducing a hybrid potential in the
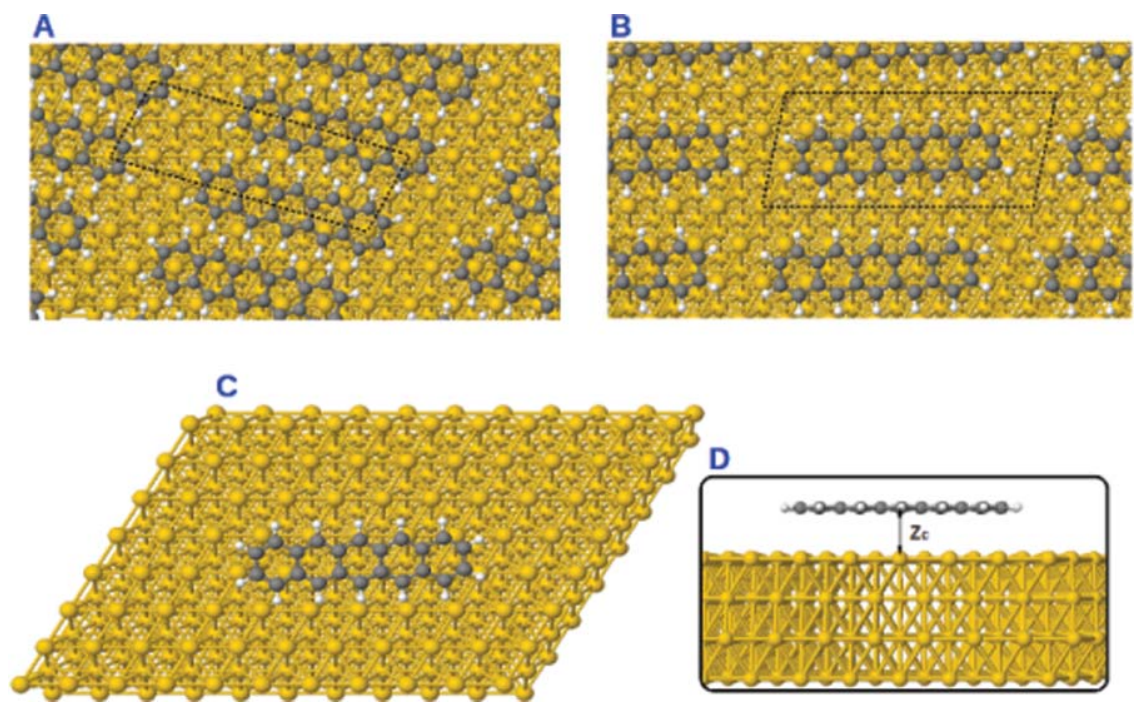

FIG. 1. Pentacene on the Au(111) surface: (a) $2 \times \sqrt{39}$ structure, (b) $6 \times \sqrt{7}$ structure, (c) cluster model, and (d) side view of a pentacene molecule on the $\mathrm{Au}(111)$ surface $\left(Z_{C}=3.2 \AA\right)$. For (a) and (b), the dashed lines denote the surface unit cells used in the calculations. 
calculations (see Sec. II B). For comparison, we have also calculated the pentacene/Au(111) interface introducing the energy gap corrections associated with Eq. (1) using a scissor Hamiltonian as discussed in Ref. 25. Obviously, $U$ depends on $E^{t}$, and this forces us to calculate $E^{t}$ and $U$ self-consistently. Both, the scissor Hamiltonian or the hybrid potential are also used to correct the error in the LDA gap due to the basis set.

(c) In the FIREBALL approach, a self-consistent version of the so-called Harris-Foulkes functional is used; in this approximation, the Kohn-Sham potential is calculated by approximating, in a self-consistent fashion, the total input charge by a superposition of spherical charges around each atom. This means that some effects like the pillow dipole (that arises due to the orthogonalization of metal and organic molecule orbitals) ${ }^{14}$ and the metalsurface dipole ${ }^{25}$ are not correctly taken into account. We have added corrections associated with these effects as perturbative corrections to our self-consistent DFT calculations. The small value of these corrections (and the tendency of both effects to cancel each other) justifies this perturbative approach.

(d) As a LDA-DFT approach does not properly take into account weak London dispersion forces, we have analyzed the metal/organic interaction correcting our LDA calculation by including van der Waals forces, using the same approach discussed in Ref. 25. This approach is based on the $L C A O-S^{2}+v d W$ formalism (LCAO stands for "linear combination of atomic orbitals") published in Refs. 35 and 36, which calculates the metal/organic interaction as the sum of two contributions: a weak chemical interaction (WCI) and the long-range van der Waals attraction. The weak chemical interaction is approximated in the present calculations by means of a corrected LDA calculation, in which the exchangecorrelation energy is calculated neglecting the effect of the overlap of the metal and organic electron densities. ${ }^{35}$ This is similar to other approaches, ${ }^{39,40}$ where a corrected short-range local correction energy is used. Regarding the van der Waals part, we have used a typical atom-atom interaction with the standard form $f_{D}(R) C_{6} / R^{6}$ ( $R$ is the distance between atoms); for $C_{6}$ we follow Ref. 25, and for $f_{D}(R)$ we use Grimme's approximation. ${ }^{25,37}$ The results of these calculations for an isolated molecule are presented in Fig. 2. The initial standard FIREBALL calculation (black curve) gives a binding energy of around $0.40 \mathrm{eV}$ and an equilibrium distance of $3.1 \AA$. In a second step, we calculate the WCI (thin red curve) and add the $C_{6} / r^{6}$-like van der Waals energy (thin green curve) to obtain the total energy (thick green curve). Therefore, we obtain an equilibrium distance of around $3.2 \AA$, similar to the one found in other calculations, ${ }^{20}$ and a binding energy of 1.8 $\mathrm{eV}$ per molecule. This energy overestimates by $\sim 0.7 \mathrm{eV}$ the experimental value of $\sim 1.1 \mathrm{eV},{ }^{38}$ probably due to the approximation used for the factor $f_{D}(R){ }^{42}$ This is the line with a recent analysis of the semiempirical van der Waals forces for azobenzene on $\operatorname{Ag}(111):{ }^{42}$ these authors have concluded, within the Tkatchenko-Scheffler (TS)-

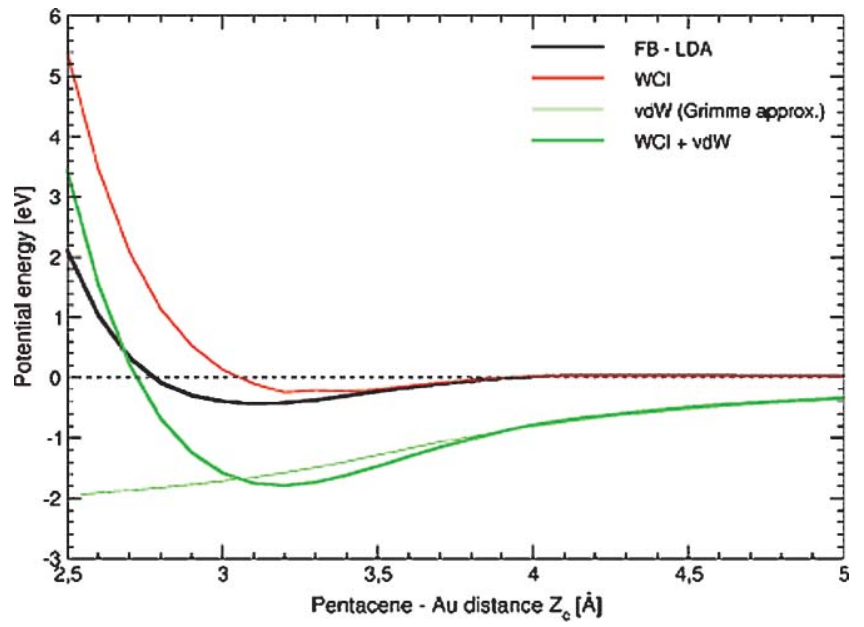

FIG. 2. Energy vs distance for the pentacene/Au(111) interaction. Black line: standard LDA-FIREBALL (FB-LDA) calculation. Red line: Weak chemical interaction calculated as discussed in the text. Thin green line: Grimme parametrization of the van der Waals interaction. Thick green line: Total pentacene-Au interaction $(\mathrm{WCI}+\mathrm{vdW})$.

semiepirical approach, ${ }^{41}$ that the semiempirical van der Waals potential tends to overestimate adsorption energies; the molecule/metal distance seems, however, to be accurately provided by these methods. In this paper, we have focused on the properties of the interface electronic structure, where the pentacene-Au distance is the relevant quantity that we take to be $3.2 \AA$.

\section{B. Hybrid functional}

Here, we discuss how we can introduce in our localorbital calculations a hybrid potential, that introduces a fraction of HF exchange potential, to get a transport energy gap satisfying Eq. (1). The main problem with this approach is that, at MO interfaces, it is not easy to have an appropriate hybrid potential for both the molecule and the metal. ${ }^{43}$ The advantage of using a local orbital basis, as done in our calculation, is that one can more easily combine hybrid potentials which are valid for both materials.

It is convenient to discuss at this point how one can introduce, in a local-orbital basis set, a Hartree-Fock approximation for the molecule. ${ }^{22}$ For this purpose, the following manybody Hamiltonian for the organic molecule is introduced: ${ }^{44}$

$$
\begin{aligned}
\hat{H}= & \sum_{i \alpha \sigma} E_{i \alpha \sigma} \hat{n}_{i \alpha \sigma}+\sum_{i \alpha \neq j \beta, \sigma} T_{i \alpha, j \beta, \sigma} \hat{c}_{i \alpha \sigma}^{+} \hat{c}_{j \beta \sigma} \\
& +\frac{1}{2} \sum_{i, \alpha \sigma \neq \beta \sigma^{\prime}} U_{i, \alpha \beta} \hat{n}_{i \alpha \sigma} \hat{n}_{i \beta \sigma^{\prime}} \\
& +\frac{1}{2} \sum_{i \neq j, \alpha \beta \sigma \sigma^{\prime}} J_{i \alpha, j \beta} \hat{n}_{i \alpha \sigma} \hat{n}_{j \beta \sigma^{\prime}},
\end{aligned}
$$

where $\hat{n}_{i \alpha \sigma}=\hat{c}_{i \alpha \sigma}^{+} \hat{c}_{i \alpha \sigma}$ and $\hat{n}_{i \alpha, j \beta \sigma}=\hat{c}_{i \alpha \sigma}^{+} \hat{c}_{j \beta \sigma}$ define the density matrix operator with mean values $n_{i \alpha \sigma}$ and $n_{i \alpha, j \beta \sigma}$, respectively; $E_{i \alpha \sigma}$ is the one electron contribution to the energy of the orbital $i \alpha \sigma$ and $T_{i \alpha, j \beta, \sigma}$ is the hopping between orbitals 
$i \alpha \sigma$ and $j \beta \sigma$. In Eq. (2), $U_{i, \alpha \beta}$ and $\left(J_{i \alpha, j \beta}\right)$ are intra-atomic (interatomic) electron-electron integrals which represent the most important contributions to the two body interaction (other small contributions are neglected in this argument). ${ }^{14,44}$ The exchange energy associated with Hamiltonian (2) can be written as follows: ${ }^{44}$

$$
\begin{aligned}
E^{x} & =-1 / 2 \sum_{i \alpha \beta \sigma} U_{i, \alpha \beta} n_{i \alpha \beta \sigma}^{2}-1 / 2 \sum_{i \alpha, j \beta \sigma} J_{i \alpha, j \beta} n_{i \alpha j \beta \sigma}^{2} \\
& \approx-1 / 2 \sum_{i \alpha \sigma} J_{i \alpha}^{e f f} n_{i \alpha \sigma}\left(1-n_{i \alpha \sigma}\right)
\end{aligned}
$$

$J_{i \alpha}^{e f f}$ being the mean interaction between the charge $n_{i \alpha \sigma}$ and its hole $\left(1-n_{i \alpha \sigma}\right)$. In our local-orbital formulation of DFT, ${ }^{4,46}$ the charges $n_{i \alpha \sigma}$ can be considered as the independent variables of the total energy, $E\left[n_{i \alpha \sigma}\right]$, in such a way that the "local" exchange potential associated with $E^{x}$ is given by

$$
\begin{aligned}
& V_{i \alpha \sigma}^{x(L)}=\delta E^{x} / \delta n_{i \alpha \sigma}, \\
& V_{i \alpha \sigma}^{x(L)}=-J_{i \alpha}^{e f f}\left(1 / 2-n_{i \alpha \sigma}\right) .
\end{aligned}
$$

As in a typical Kohn-Sham-like approach, the charges $n_{i \alpha \sigma}$ are calculated self-consistently using the effective Hamiltonian,

$$
\hat{H}^{e f f}=\sum_{i \alpha \sigma} \varepsilon_{i \alpha \sigma} \hat{n}_{i \alpha \sigma}+\sum_{i \alpha \neq j \beta, \sigma} T_{i \alpha, j \beta, \sigma} \hat{c}_{i \alpha \sigma}^{+} \hat{c}_{j \beta \sigma},
$$

with $\varepsilon_{i \alpha \sigma}=E_{i \alpha \sigma}+V_{i \alpha \sigma}^{H}+V_{i \alpha \sigma}^{x}$, where the Hartree and local exchange potential have been introduced (in this argument we have neglected electron correlation effects). ${ }^{44}$

The problem of underestimation of the gap in local density theories (like standard LDA or the one presented above) can be avoided by using a Hartree-Fock approximation for the treatment of the exchange energy. In our particular case, the idea is to use for the exchange energy the expression ${ }^{44}$

$$
\begin{aligned}
E^{x}= & -1 / 2 \sum_{i \alpha \beta \sigma} U_{i, \alpha \beta} n_{i \alpha \beta \sigma} n_{i \beta \alpha \sigma} \\
& -1 / 2 \sum_{i \alpha, j \beta \sigma} J_{i \alpha, j \beta} n_{i \alpha j \beta \sigma} n_{j \beta i \alpha \sigma},
\end{aligned}
$$

and introduce in a HF-approximation the following offdiagonal exchange potential terms:

$$
\begin{aligned}
V_{i \alpha \beta \sigma}^{\mathrm{HF}} & =-U_{i, \alpha \beta} n_{i \beta \alpha \sigma}, \\
V_{i \alpha, j \beta \sigma}^{\mathrm{HF}} & =-J_{i \alpha, j \beta \sigma} n_{j \beta, i \alpha \sigma} .
\end{aligned}
$$

In this approach, the charges $n_{i \alpha \sigma}$ are calculated selfconsistently using the effective Hamiltonian,

$$
\hat{H}^{\prime e f f}=\sum_{i \alpha \sigma} \varepsilon_{i \alpha \sigma}^{\prime} \hat{n}_{i \alpha \sigma}+\sum_{i \alpha \neq j \beta, \sigma} T_{i \alpha, j \beta, \sigma}^{\prime} \hat{c}_{i \alpha \sigma}^{+} \hat{c}_{j \beta \sigma},
$$

where $\varepsilon_{i \alpha \sigma}^{\prime}=E_{i \alpha \sigma}+V_{i \alpha \sigma}^{H}$ includes the Hartree approximation to the orbital levels and $T_{i \alpha j \beta \sigma}^{\prime}=T_{i \alpha j \beta \sigma}+V_{i \alpha j \beta \sigma}^{H F}$ is the Fock terms. Notice that, while in Eq. (5) the exchange energy contributes with a diagonal term to the energy levels, in Eq. (8) the exchange contributions appear as off-diagonal terms in the hopping. In this HF approximation, ${ }^{22}$ the energy levels of the effective Hamiltonian (8) yield a transport energy gap larger than the one calculated from Hamiltonian (5); in particular, this HF energy gap for the molecule is much closer to the experimental one and, in some way, embodies the major part of the effect associated with $U_{0}$ in LDA $\left(U_{0}\right.$ is the value of $U$ for the gas-phase molecule).

In our approach to the organic molecule/metal interface, we combine Eqs. (3) and (6) and write the exchange energy for the molecule local orbitals, $i \alpha \sigma$, as follows:

$$
\begin{aligned}
E_{m o l}^{x}= & -(1-\beta) / 2 \sum_{i \alpha \sigma} J_{i \alpha}^{e f f} n_{i \alpha \sigma}\left(1-n_{i \alpha \sigma}\right) \\
& -\beta / 2 \sum_{i \alpha \beta \sigma} U_{i, \alpha \beta} n_{i \alpha \beta \sigma} n_{i \beta \alpha \sigma} \\
& -\beta / 2 \sum_{i \alpha, j \beta \sigma} J_{i \alpha, j \beta} n_{i \alpha j \beta \sigma} n_{j \beta i \alpha \sigma},
\end{aligned}
$$

where $\beta$ is a parameter that defines how much exchange energy is treated in a local approximation,

$$
E^{x(L)}=-(1-\beta) / 2 \sum_{i \alpha \sigma} J_{i \alpha}^{e f f} n_{i \alpha \sigma}\left(1-n_{i \alpha \sigma}\right),
$$

as a local potential $V_{i \alpha}^{x(L)}=-(1-\beta) J_{i \alpha}^{e f f}\left(1 / 2-n_{i \alpha \sigma}\right)$, and how much is treated in $\mathrm{HF}$,

$$
\begin{aligned}
E^{x(\mathrm{HF})}= & -\beta / 2 \sum_{i \alpha \beta \sigma} U_{i, \alpha \beta} n_{i \alpha \beta \sigma} n_{i \beta \alpha \sigma} \\
& -\beta / 2 \sum_{i \alpha, j \beta \sigma} J_{i \alpha, j \beta} n_{i \alpha j \beta \sigma} n_{j \beta i \alpha \sigma},
\end{aligned}
$$

as an off-diagonal term $V_{i \alpha \beta \sigma}^{\mathrm{HF}}(\beta)=-\beta U_{i, \alpha \beta} \underline{\mathrm{n}}_{i \beta \alpha \sigma}$; $V_{i \alpha, j \beta \sigma}^{\mathrm{HF}}(\beta)=-\beta J_{i \alpha, j \beta \sigma} n_{j \beta, i \alpha \sigma}$.

These ideas can be used in a practical way to include a fraction $\beta$ of $\mathrm{HF}$ exchange potential for the molecule (but not the metal) in our local-orbital LDA calculations. This is done introducing the corresponding off-diagonal terms in the molecule, $V_{i \alpha \beta \sigma}^{\mathrm{HF}}(\beta)$ and $V_{i \alpha, j \beta \sigma}^{\mathrm{HF}}(\beta)$, subtracting at the same time in the same orbitals, $i \alpha \sigma$, the corresponding local exchange potential $-\beta J_{i \alpha}^{\text {eff }}\left(1 / 2-n_{i \alpha \sigma}\right)$. It should be stressed that the role of $\beta$ is to fix the transport energy gap of the molecule to the self-consistent value given by Eq. (1). In a sense, this hybrid potential plays a role similar to the one played by the scissor operator discussed in Ref. 25. In a similar way, $\beta$ is adjusted to get consistency between the values of $E^{t}$ and $U$.

\section{DENSITY OF STATES, INTERFACE DIPOLE, AND CHARGING ENERGY}

\section{A. Hybrid potential results}

In a first step, we present calculations for pentacene/Au(111) using the hybrid potential we have just discussed. Figure 3 shows our calculated DOS projected on the molecular orbitals for the three cases we have considered: (1) a single molecule (c), (2) the $6 \times \sqrt{7}$ adlayer (b), and (3) the $2 \times \sqrt{39}$ monolayer (a) (see Fig. 1).

In these figures, we have also shown the molecular levels of the isolated molecule (in green), with the transport energy gap, $E^{t}=3.1 \mathrm{eV}$, as calculated using the self-consistent hybrid potential with $\beta=0.31$; in this consistency, we have also 

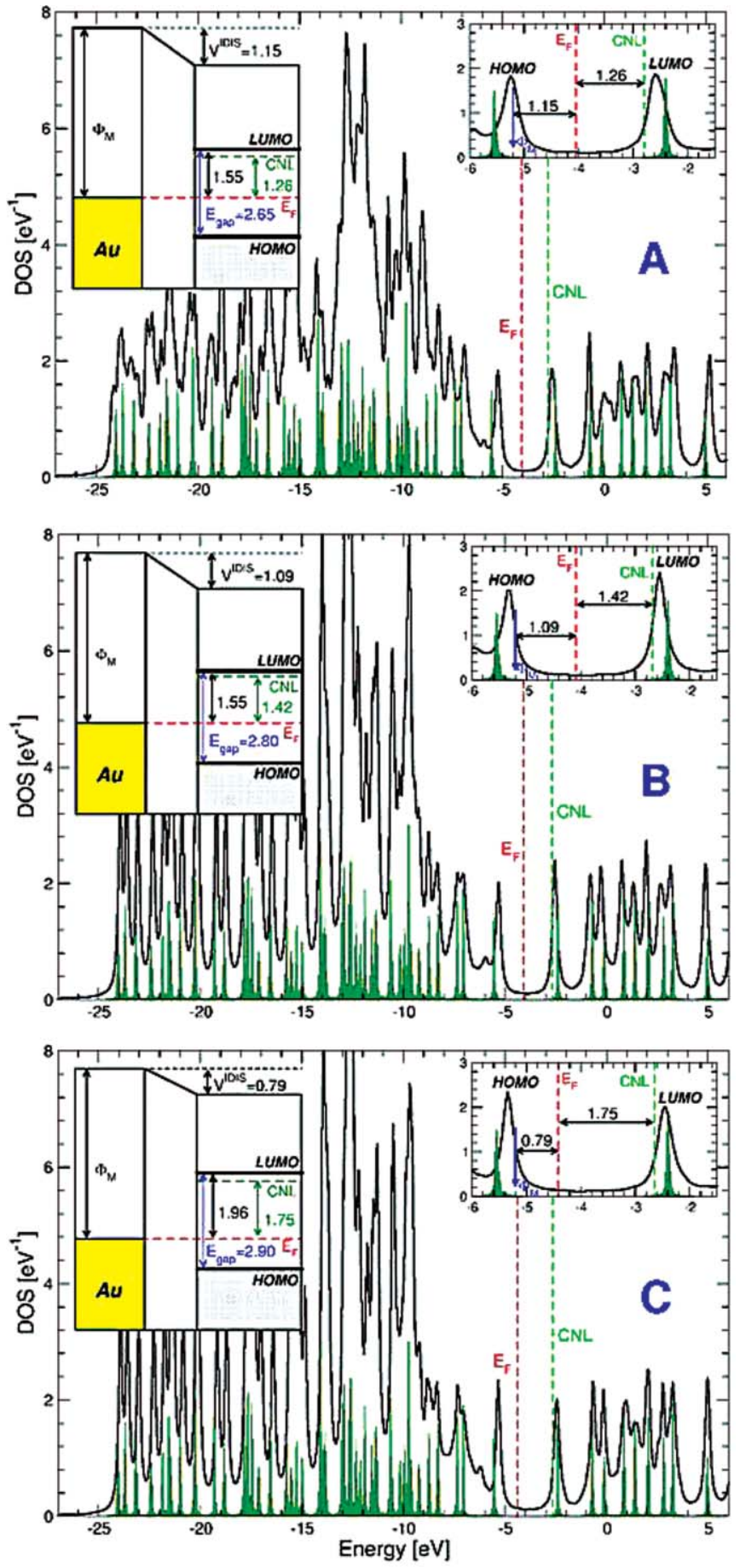

FIG. 3. DOS (obtained with the hybrid potential) projected on the pentacene orbitals for the geometries of Fig. 1 (case (a): $2 \times \sqrt{39}$ structure, case (b): $6 \times \sqrt{7}$ structure, and case (c): cluster model). CNL of the molecule, metal work-function, and Fermi level of the system are also shown. Molecular levels of the isolated molecule, calculated with the same hybrid potential, are indicated in green. Right inset: enlarged image for the area around the HOMO and LUMO levels. Left inset: interface levels scheme. All values are given in $\mathrm{eV}$.

included the metal surface dipole as explained below, an effect that reduces the transport energy gap by $0.2 \mathrm{eV}$. Notice that this energy gap is a little larger than the peak-to-peak gap obtained from the calculated DOS; in particular, $E^{t}=2.65$, 2.8 , and $2.9 \mathrm{eV}$ for cases (a), (b), and (c), respectively. In each figure, the HOMO and LUMO levels, the molecule charge neutrality level, and the final Fermi energy, $E_{F}$, are shown; notice that the CNL (calculated integrating the DOS up to charge neutrality conditions in the molecule) is located in all the cases around $\sim 0.2 \mathrm{eV}$ from the LUMO peak (some fluctuations around this value are probably related to the precision of our calculations). On the left insets, we see how the metal Fermi energy and the organic levels are aligned; notice that the molecular levels are shifted with respect to the metal Fermi energy by a potential, $V^{\text {IDIS }}$, that takes the values: $V^{\mathrm{IDIS}}=1.15 \mathrm{eV}(\mathrm{a}), 1.09 \mathrm{eV}$ (b), and $0.79 \mathrm{eV}$ (c), decreasing from the compact monolayer (a), towards the $6 \times \sqrt{7}$ structure (b) and the single molecule case (c). These results, and the mechanism controlling this level alignment, can be understood in terms of the IDIS model (and the charge transfer mechanism) which relates the band alignment to the charge transfer.

Figure 4 shows, for the cases (a), (b), and (c), $V^{\mathrm{IDIS}}$ and the charge transfer, $\delta n$, as a function of a fictitious metal work-function which is introduced by means of the shift operator discussed above. The crucial point to realize in this figure is how $V^{\mathrm{IDIS}}$ and $\delta n$ depend linearly on metal workfunction, $-\Phi_{M}$, and how $V^{\mathrm{IDIS}} \cong 0$ when $\delta n=0$. This shows that $V^{\mathrm{IDIS}}$ is associated with the charge transfer between the metal and the organic molecule; then, following the IDIS model, ${ }^{22,25}$ we can write

$$
V^{\mathrm{IDIS}}=(1-S)\left(\mathrm{CNL}-\Phi_{M}\right),
$$

where $\Phi_{M}$ is the initial metal Fermi level and $S$ is the screening parameter. Notice that Eq. (10) implies that

$$
\left(E_{F}-\Phi_{M}\right)=S\left(\mathrm{CNL}-\Phi_{M}\right) .
$$

From Fig. 4, we find $S=0.52$ (a), 0.57 (b), and 0.69 (c), indicating that in the compact structure screening effects are the largest, and in the single molecule the smallest $(S \rightarrow 0$ for large screening, and $S \rightarrow 1$ for a small one).

As mentioned above, we introduce the pillow dipole and the metal-surface dipole as a small correction to the previous calculations. The pillow dipole is calculated following Ref. 25; in the minimal basis set used in our calculations, that pillow dipole is negligible. We have found, however, that using a more extended basis set $\left(s p^{3} d^{5} s^{*} d^{* 5}\right.$ for $\mathrm{Au}$, $s p^{3} d^{5}$ for $\mathrm{C}$, and $s s^{*}$ for $\mathrm{H}$; see Ref. 25 for details) yields a bare pillow dipole potential, $e V_{0}^{P}$, of $0.5 \mathrm{eV}$ for $2 \times \sqrt{39}$ monolayer. Screening effects reduce this potential to $e V^{P}$ $=e S V_{0}^{P}=0.25 \mathrm{eV}$; this pillow potential should be added to $V^{\text {IDIS }}$, yielding a total molecular potential, $V^{t}$, given by $V^{t}$ $=V^{\mathrm{IDIS}}+V^{P}$.

On the other hand, the metal surface dipole correction is calculated using the off-diagonal elements of the Green functions as afforded by the tight-binding Hamiltonian obtained from our hybrid-DFT calculation (see Ref. 25). In the tightpacked $2 \times \sqrt{39}$ monolayer, we find that $\left(E_{F}-\Phi_{M}\right)$ is reduced by $0.15 \mathrm{eV}$, this effect compensating to a large extent the potential, $V^{P}$, created by the pillow effect. For the single molecule case, we find that this compensation is even better; this metal surface dipole correction reduces, however, $U$ and the transport energy gap ${ }^{25}$ by $0.2 \mathrm{eV}$, as stated before. 


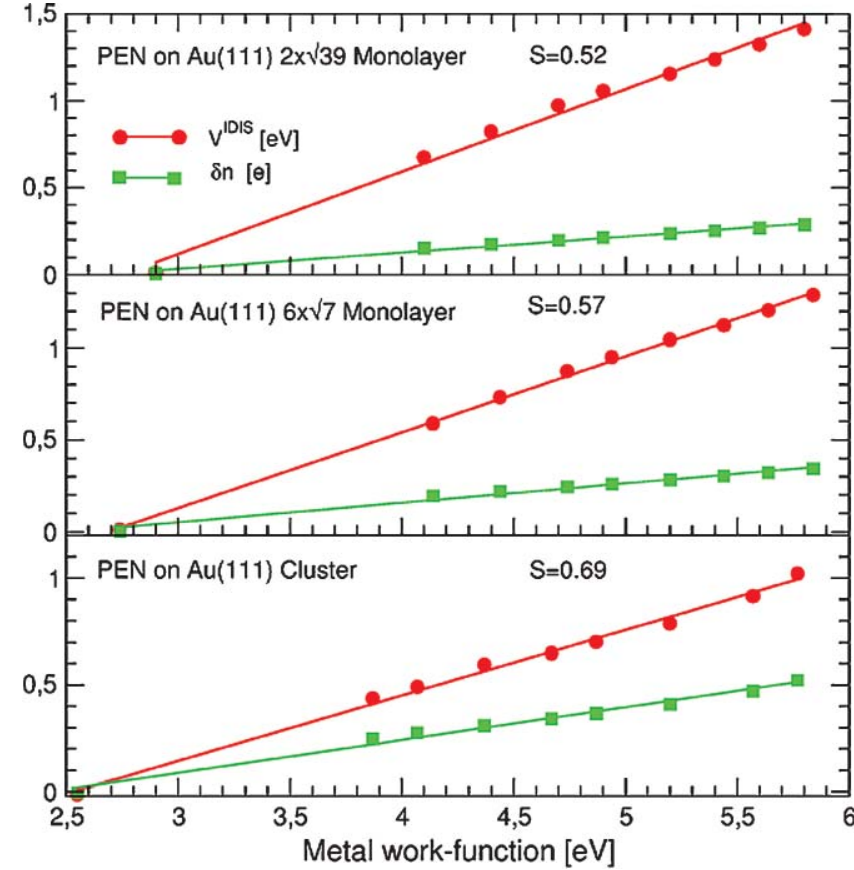

FIG. 4. $V^{\mathrm{IDIS}}$ and $\delta n$ - charge transfer from the molecule to the metal (obtained with the hybrid potential) as a function of the initial Fermi Level. This change in the initial Fermi level (that can be considered as the metal work function with a minus sign), tries to simulate in an approximate way how the interface properties depend on the different metals.

\section{B. LDA with a scissor operator results}

We have also calculated the pentacene/Au(111) interface using a scissor operator to correct the transport gap using Eq. (1), instead of the hybrid potential, in order to compare both approaches and see the difference that this simpler approximation introduces in the bands alignment and in the molecule energy gap. Figure 5 shows the DOS projected on the molecular orbitals for the same cases shown in Fig. 3; notice that the calculated DOS using the scissor operator is very similar to the one obtained with the hybrid potential. There are, however, some relevant differences, because although $V^{\mathrm{IDIS}}$ is the same in both cases, we find an important change in the molecule energy gap; while for the hybrid potential case we find for the isolated molecule $E^{t}=3.1 \mathrm{eV}$, using the scissor operator yields $E^{t}=3.4 \mathrm{eV}$, so that the peak-to-peak energy gap is $E^{t}=3.05$ (a), 3.2 (b), and $3.15 \mathrm{eV}$ (c). The important point to realize about these results is that the calculated energy gap for the molecule, using the hybrid potential, is $0.3 \mathrm{eV}$ smaller than the one calculated using the scissor operator.

We believe this difference is due to the delocalization of the intra-molecular exchange hole over the metal: for the isolated molecule, one can think of the self-interaction correction (or the charging energy, $U_{0}$ ) as being created by the exchange hole that eliminates the interaction of the molecular charge with itself; introducing the metal-organic interaction delocalizes that hole and reduces, in our Hartree-Fock calculation, the self-interaction correction. We have found that, due to this pentacene/Au interfaction, around $8 \%$ of the molecular exchange hole is delocalized into the metal.
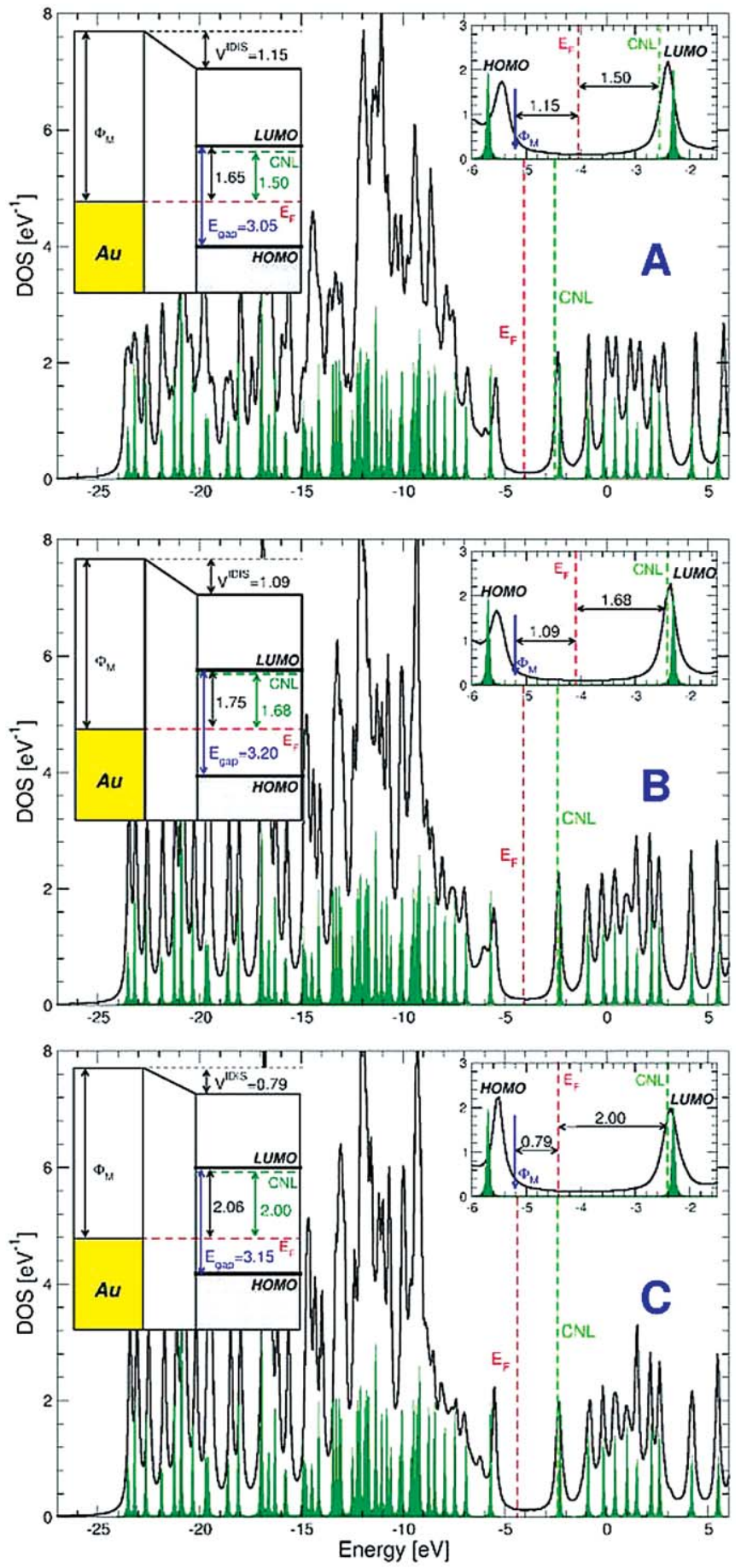

FIG. 5. DOS (obtained with the scissor operator) projected on the pentacene orbitals for the geometries of Fig. 1 (case (a): $2 \times \sqrt{39}$ structure, case (b): $6 \times \sqrt{7}$ structure, and case (c): cluster model). CNL of the molecule, metal work-function, and Fermi level of the system are also shown. Molecular levels of the isolated molecule are indicated in green. Right inset: Enlarged image for the area around the HOMO and LUMO levels. Left inset: Interface levels scheme. All values are given in $\mathrm{eV}$.

Figure 6 shows $V^{\text {IDIS }}$ and $\delta n$ as a function of the fictitious metal work-function. This figure also confirms the linearity between $V^{\mathrm{IDIS}}$ and $\delta n$, and how much appropriate the IDIS model is to explain the band alignment between $\mathrm{Au}$ and pentacene. 


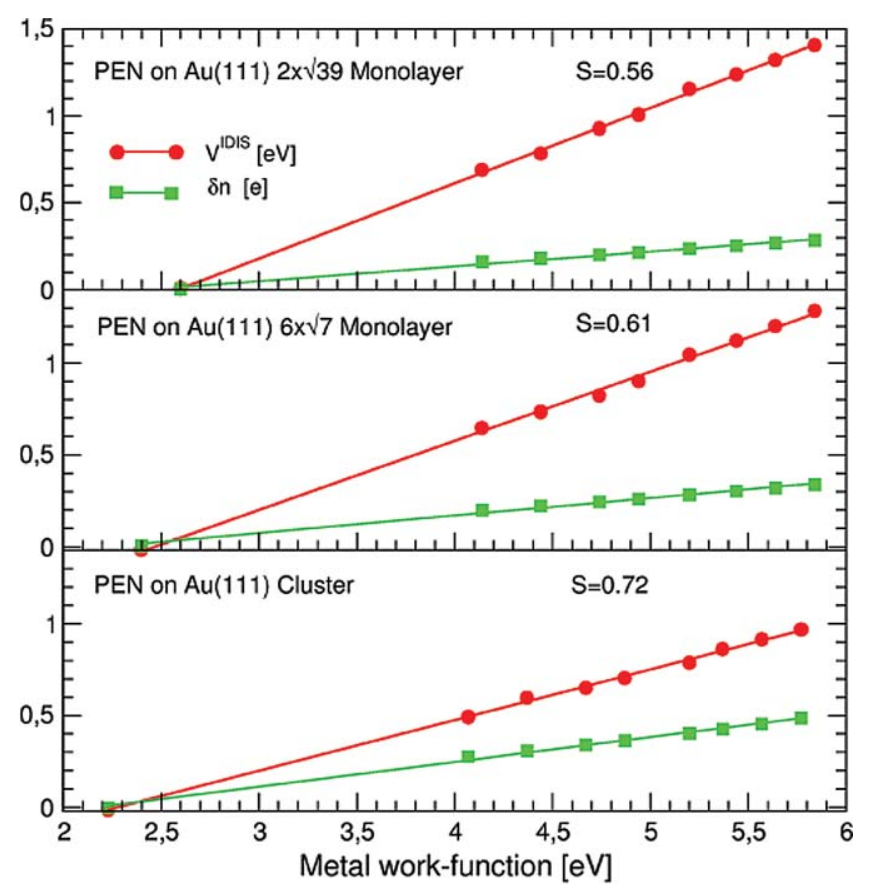

FIG. 6. $V^{\text {IDIS }}$ and $\delta n$ - charge transfer from the molecule to the metal (obtained with the scissor operator) as a function of the initial Fermi level. This change in the initial Fermi level (that can be considered as the metal work function with a minus sign), tries to simulate in an approximate way how the interface properties depend on the different metals.

Regarding the pillow dipole and the metal-surface dipole corrections, we only mention here that we find that our results are very similar to the ones calculated using the hybrid potential approach.

\section{DISCUSSION AND CONCLUSIONS}

We have presented a hybrid-DFT calculation for pentacene on $\mathrm{Au}(111)$ considering the following cases: a single molecule, a $6 \times \sqrt{7}$ adlayer, and a $2 \times \sqrt{39}$ monolayer. Our approach is similar to the one already published for benzene/Au(111) ${ }^{25}$ except for the new hybrid functional discussed in Sec. II B. This hybrid potential is introduced to fix the transport energy gap, $E^{t}$, to the value given by Eq. (1), with the molecule charging energy, $U$, calculated by means of the IDIS potential, $V^{\text {IDIS }}$, and the charge transfer between the metal and the molecule. We have also analyzed the pentacene/Au interface using a scissor operator as done for benzene. It is interesting to stress that the results of both approaches, the hybrid potential and the scissor operator, are similar, showing that the interface barrier height is basically controlled by the charge transfer between the two materials; the only significant difference is found for transport energy gap, $E^{t}$, that is around $0.35 \mathrm{eV}$ smaller in the case of the hybrid potential. We interpret that result as due to the delocalization in the metal of the exchange-hole associated with the self-interaction correction. ${ }^{22}$ The values found for $E^{t}$ in the $2 \times \sqrt{39}$ structure are $2.65 \mathrm{eV}$ (hybrid potential) and $3.05 \mathrm{eV}$ (scissor operator); theses quantities can be compared with the experimental data of Amy et al. ${ }^{47}$ who have mea- sured $E^{t}=2.88 \mathrm{eV}$ for a pentacene thickness of $20 \AA$, in good agreement with our hybrid-DFT calculations (one can expect $E^{t}$ to be slightly smaller than $2.88 \mathrm{eV}$ for a pentacene monolayer). ${ }^{47}$

Regarding the metal work-function change due to the pentacene deposition, we have found in our calculations that the $6 \times \sqrt{7}$ and the $2 \times \sqrt{39}$ structures yield very similar results. In both cases, the interface electrostatic dipole $\Delta=4 \pi \frac{d \delta n}{A}$ ( $d$ is the effective distance between the charges of the molecule and the metal, $A$ is the area per molecule), coincides practically with $V^{\text {IDIS }} 1.09 \mathrm{eV}$ and $1.15 \mathrm{eV}$ for the (b) and (a) structures, respectively. The experimental evidence ${ }^{19,48,49}$ indicates that this interface dipole is $0.95 \mathrm{eV}$, in good agreement with our results. Morikawa et al. ${ }^{20}$ have calculated an interface dipole of $1.19 \mathrm{eV}$ for a $6 \times \sqrt{7}$ pentacene/ $\mathrm{Au}(111)$ structure and a pentacene-Au distance of $3.2 \AA$; the interface dipole and the metal/organic distance are both in good agreement with our calculations.

It is also important to discuss briefly, following Ref. 25, the issue of the convergence of our calculations with the basis set. As mentioned in that reference, using a more complete basis set changes significantly the molecular levels; however, the shift operator and the hybrid potential (or the scissor operator) used in our calculations allow us to fit the molecule energy gap and its energy position to the experimental values: these corrections make our calculations of the charging energy and the interface dipole reasonably well-converged quantities (this is checked independently by the good agreement found with the calculations of Morikawa et al.). ${ }^{20}$ An exception to this conclusion is the calculation of the pillow dipole that we find to depend largely on the atomic basis set; as discussed in Sec. III A, for an extended basis set the pillow potential for a full monolayer is $0.25 \mathrm{eV}$, a value that is largely compensated by the surface dipole correction. This suggests that our calculated change of the metal work-function for the $6 \times \sqrt{7}$ and the $2 \times \sqrt{39}$ structures has an error bar of $0.1 \mathrm{eV}$.

In conclusion, we have presented a hybrid-DFT calculation of the interface properties of the pentacene/Au(111) interface, including a self-consistent calculation of the molecule charging energy and its transport energy gap. Our results show a good agreement with the experimental data for the transport energy gap and the metal work-function change for a monolayer coverage with another theoretical calculation. This lends strong support to our interpretation of this metal/organic level alignment due to the charge transfer between the metal and pentacene, as described in the IDIS model.

\section{ACKNOWLEDGMENTS}

This work is supported by the Spanish MICIIN under Contract No. FIS2010-16046, the CAM under Contract No. S2009/MAT-1467, and the European Project MINOTOR (Grant No. FP7-NMP-228424). B.P. gratefully acknowledges financial support by the CAM, and E.A. acknowledges financial support by the CAM, the FSE, and the European Project MINOTOR. 
${ }^{1}$ X. L. Chen, A. J. Lovinger, Z. Bao, and J. Sapjeta, Chem. Mater. 13, 1341 (2001).

${ }^{2}$ W. R. Salanek, S. Stafstrom, and J. L. Bredas, Conjugated Polymer Surfaces and Interfaces: Electronic and Chemical Structure of Interfaces for Polymer Light emitting Diodes (Cambridge University Press, Cambridge, England, 1996).

${ }^{3}$ N. Koch, ChemPhysChem 8, 1438 (2007).

${ }^{4}$ S. Narioka, H. Ishii, D. Yoshimura, M. Sei, Y. Ouchi, K. Seki, S. Hasegawa, T. Miyazaki, Y. Harima, and K. Yamashita, Appl. Phys. Lett. 67, 1899 (1995).

${ }^{5}$ I. G. Hill, A. Rajagopal, A. Kahn, and Y. Hu, Appl. Phys. Lett. 73, 662 (1998).

${ }^{6}$ H. Ishii, K. Sugiyama. E. Ito, and K. Seki, Adv. Mater. 11, 605 (1999).

${ }^{7}$ I. G. Hill, J. Schwartz, and A. Kahn, Org. Electron. 1, 5 (2000).

${ }^{8}$ C. Shen and A. Kahn, Org. Electron. 2, 89 (2001).

${ }^{9}$ X. Crispin, V. Geskin, A. Crispin, J. Cornil, R. Lazzaroni, W. R. Salaneck, and J. L. Bredas, J. Am. Chem. Soc. 124, 8131 (2002).

${ }^{10}$ M. Knupfer and G. Paasch, J. Vac. Sci. Technol. A 23, 1072 (2005).

${ }^{11}$ S. Yanagisawa and Y. Morikawa, J. Phys.: Condens. Matter 21, 064247 (2009).

${ }^{12}$ P. S. Bagus, V. Staemmler, and C. Woell, Phys. Rev. Lett. 89, 096104 (2002).

${ }^{13}$ G. Witte, S. Lukas, P. S. Bagus, and C. Woell, Appl. Phys. Lett. 87, 263502 (2005).

${ }^{14}$ H. Vazquez, Y. J. Dappe, J. Ortega, and F. Flores, J. Chem. Phys. 126, 144703 (2007).

${ }^{15}$ H. Vazquez, R. Oszwaldowski, P. Pou, J. Ortega, R. Perez, F. Flores, and A. Kahn, Europhys. Lett. 65, 802 (2004).

${ }^{16}$ H. Vazquez, F. Flores, R. Oszwaldowski, J. Ortega, R. Perez, and A. Kahn, Appl. Surf. Sci. 234, 108 (2004).

${ }^{17}$ M. G. Betti, A. Kanjilal, C. Mariani, H. Vazquez, Y. J. Dappe, J. Ortega, and F. Flores, Phys. Rev. Lett. 100, 027601 (2008).

${ }^{18}$ M. Fahlman, A. Crispin, X. Crispin, S. K. M. Henze, M. P. de Jong, W. Osikowicz, C. Tengstedt, and W. R. Salaneck, J. Phys.: Condens. Matter 19, 183202 (2007).

${ }^{19}$ P. G. Schroeder, C. B. France, J. B. Park, and B. A. Parkinson, J. Appl. Phys. 91, 3010 (2002).

${ }^{20}$ K.Toyoda, I. Hamada, K. Lee, S. Yanagisawa, and Y. Morikawa, J. Chem. Phys. 132, 134703 (2010)

${ }^{21}$ S. Kummeland and L. Kronik, Rev. Mod. Phys. 80, 3 (2008).

${ }^{22}$ F. Flores, J. Ortega, and H. Vazquez, Phys. Chem. Chem. Phys. 11, 8658 (2009).

${ }^{23}$ E. Abad, C. Gonzalez, J. Ortega, and F. Flores, Org. Electron. 11, 332 (2010).

${ }^{24}$ J. D. Sau, J. B. Neaton, H. J. Choi, S. G. Louie, and M. L. Cohen, Phys. Rev. Lett. 101, 026804 (2008).
${ }^{25}$ E. Abad, Y. J. Dappe, J. I. Martinez, F. Flores, and J. Ortega, J. Chem. Phys. 134, 044701 (2011).

${ }^{26}$ P. Jelinek, H. Wang, J. P. Lewis, O. F. Sankey, and J. Ortega, Phys. Rev. B 71, 235101 (2005); J. P. Lewis, K. R. Glaesemann, G. A. Voth, J. Fritsch, A. A. Demkov, J. Ortega, and O. F. Sankey, ibid. 64, 195103 (2001).

${ }^{27}$ O. F. Sankey and D. J. Niklewski, Phys. Rev. B 40, 3979 (1989).

${ }^{28}$ A. A. Demkov, J. Ortega, O. F. Sankey, and M. P. Grumbach, Phys. Rev. B 52, 16178 (1995).

${ }^{29}$ A. Maeland and T. Flanagan, Can. J. Phys. 42, 2364 (1964).

${ }^{30}$ M. A. Basanta, Y. J. Dappe, P. Jelinek, and J. Ortega, Comput. Mater. Sci. 39, 759 (2007).

${ }^{31}$ R. B. Campbell and J. M. Robertson, Acta Crystallogr. 15, 289 (1962).

${ }^{32}$ R. G. Endres, C. Y. Fong, L. H. Yang, G. Witte, and C. Woell, Comput. Mater. Sci. 29, 362 (2004)

${ }^{33}$ N. E. Gruhn, D. A da Silva Filho, T. G. Bill, M. Malagoli, V. Coropceanu, A. Kahn, and J. L. Bredas, J. Am. Chem. Soc. 124, 7918 (2002).

${ }^{34}$ N. Sato, H. Inokuchi, and E. A. Silinsh, Chem. Phys. 115, 269 (1987).

${ }^{35}$ Y. J. Dappe, M. A. Basanta, J. Ortega, and F. Flores, Phys. Rev. B 74, 205434 (2006).

${ }^{36}$ Y. J. Dappe, J. Ortega, and F. Flores, Phys. Rev. B 79, 165409 (2009).

${ }^{37}$ S. Grimme, J. Antony, T. Schwabe, and C. Mueck-Lichtenfeld, Org. Biomol. Chem. 5, 741 (2007); S. Grimme, J. Comput. Chem. 27, 1787 (2006).

${ }^{38}$ C. B. France, P. G. Schroeder, J. C. Forsythe, and B. A. Parkinson, Langmuir 19, 12 (2003).

${ }^{39}$ G. Murdachaew, S. de Gironcoli, and G. Scoles, J. Phys. Chem. A 112, 9993 (2008).

${ }^{40}$ K. Pernal, R. Podeszwa, K. Patkowski, and K. Szalewicz, Phys. Rev. Lett. 103, 263201 (2009).

${ }^{41}$ A. Tkatchenko and M. Scheffler, Phys. Rev. Lett. 102, 073005 (2009).

${ }^{42}$ G. Mercurio, E. R. McNellis, I. Martin, S. Hagen, F. Leyssner, S. Soubatch, J. Meyer, M. Wolf, P. Tegeder, F. S. Tautz, and K. Reuter, Phys. Rev. Lett. 104, 036102 (2010).

${ }^{43}$ J. Paier, M. Marsman, and G. Kresse, J. Chem. Phys 127, 024103 (2007).

${ }^{44}$ P. Pou, R. Perez, F. Flores, A. Levy-Yeyati, A. Martin-Rodero, J. M. Blanco, F. J. Garcia-Vidal, and J. Ortega, Phys. Rev. B 62, 4309 (2000).

${ }^{45}$ F. J. Garcia-Vidal, J. Merino, R. Perez, R. Rincon, J. Ortega, and F. Flores, Phys. Rev. B 50, 10537 (1994).

${ }^{46}$ K. Schonhammer, O. Gunnarson, and R. Noack, Phys. Rev. B 52, 2504 (1995).

${ }^{47}$ F. Amy, C. Chan, and A. Kahn, Org. Electron. 6, 85 (2005).

${ }^{48}$ X. Liu, Y Zhan, S. Braun, F. Li, and M. Fahlman, Phys. Rev. B 80, 115401 (2009).

${ }^{49}$ N. Koch, J. Phys.: Condens. Matter 20, 184008 (2008). 